\title{
Predictions of variable mass loss for Luminous Blue Variables
}

\author{
Jorick S. Vink ${ }^{1,2}$ and A. de Koter $^{3}$ \\ ${ }^{1}$ Imperial College, Blackett Laboratory, Prince Consort Road, London, SW7 2BZ, UK \\ 2 Astronomical Institute, Utrecht University, PO Box 80000, 3508 TA Utrecht, The Netherlands \\ 3 Astronomical Institute "Anton Pannekoek", University of Amsterdam, Kruislaan 403, 1098 SJ Amsterdam, The Netherlands
}

Received 16 April 2002 / Accepted 8 July 2002

\begin{abstract}
We present radiation-driven wind models for Luminous Blue Variables (LBVs) and predict their mass-loss rates. We study the effects of lower masses and modified abundances in comparison to the normal OB supergiants, and we find that the main difference in mass loss is due to the lower masses of LBVs. In addition, we find that the increase in helium abundance changes the mass-loss properties by small amounts (up to about $0.2 \mathrm{dex}$ in $\log \dot{M}$ ), while CNO processing is relatively unimportant for the mass-loss rate. A comparison between our mass loss predictions and the observations is performed for four relatively well-studied LBVs. The comparison shows that (i) the winds of LBVs are driven by radiation pressure on spectral lines, (ii) the variable mass loss behaviour of LBVs during their S Doradus-type variation cycles is explained by changes in the line driving efficiency, notably due to the recombination/ionisation of $\mathrm{Fe}$ IV/III and $\mathrm{Fe}$ III/II, and finally, (iii) the winds of LBVs can be used to derive their masses, as exemplified by the case of AGCar, for which we derive a present-day mass of $35 M_{\odot}$.
\end{abstract}

Key words. stars: early-type - stars: mass-loss - stars: supergiants - stars: winds, outflows - stars: evolution

\section{Introduction}

Luminous Blue Variables (LBVs) are massive stars in a brief $\left(\sim 10^{5} \mathrm{yr}\right)$ but violent post-main-sequence phase of evolution, during which they lose the remaining parts of their hydrogen-rich outer layers, before entering the WolfRayet stage. They show a complex pattern of visual brightness variations (Humphreys \& Davidson 1994; Lamers 1995; van Genderen 2001), but most characteristic are their visual light variations of $\Delta V \simeq 1-2$ mag on timescales of years. These changes are referred to as "typical" or "S Doradus-type" outbursts. During these variability cycles, the stars move horizontally in the Hertzsprung-Russell Diagram (HRD) as they expand in radius at approximately constant luminosity. At minimum visual brightness the star is relatively hot $\left(T_{\mathrm{eff}} \sim\right.$ $20000-30000 \mathrm{~K}$ ) and small, whereas the star is large and relatively cool $\left(T_{\text {eff }} \sim 8000 \mathrm{~K}\right)$ at visual maximum. Although it has been established that the typical variations are related to changes in stellar radius rather than the formation of a pseudophotosphere (Leitherer et al. 1989; de Koter et al. 1996), the physical reason for these radius changes - which can be as large as factors of ten - is as yet unidentified (see Nota \& Lamers 1997 for overviews).

The strong stellar winds of LBV stars $(\dot{M} \sim$ $10^{-4}-10^{-6} M_{\odot} \mathrm{yr}^{-1}$ ) show a wide variety of mass loss behaviour, which is poorly understood. There are cases like R 71 where the mass loss increases while the star expands (Leitherer et al. 1989), whereas R 110 shows a behaviour

Send offprint requests to: Jorick S. Vink, e-mail: j .vink@ic . ac . uk that is the exact opposite: while the star increases in size, its mass-loss rate drops (Stahl et al. 1990). These results imply that there is no straightforward correlation between mass loss and radius (or effective temperature) for LBVs (Leitherer 1997).

Recent radiation-driven wind models for normal $\mathrm{O}$ and B stars however show that one does not expect such a strict monotonous behaviour of mass loss with effective temperature. Vink et al. $(1999,2000)$ have found an overall trend that cooler stars - at constant luminosity - experience smaller mass loss, but at specific effective temperatures the mass-loss rate jumps upward. The overall trend can be explained by a growing mismatch between the position of the bulk of the driving lines (mostly in the ultraviolet) and the location of the flux maximum, which shifts gradually towards the optical for cooler stars. Superimposed on this there are jumps, where $\dot{M}$ increases steeply due to recombinations of dominant line-driving ions, specifically those of iron. At $T_{\text {eff }} \simeq 25000 \mathrm{~K}$, the mass loss is predicted to increase by about a factor of five due to the recombination of Fe IV to Fe III. Observational evidence for this socalled "bi-stability" jump has been presented by Lamers et al. (1995), who discovered an abrupt discontinuity in the carbon ionisation and the terminal wind velocities of stars at spectral type B1 $\left(T_{\text {eff }} \simeq 21000 \mathrm{~K}\right)$. They also present evidence for a second jump at spectral type $\mathrm{A} 0\left(T_{\text {eff }} \simeq 10000 \mathrm{~K}\right)$, which is anticipated to be due to the recombination of Fe III to Fe II (Leitherer et al. 1989).

The main goal of this paper is to investigate whether radiation pressure on spectral lines is responsible for the mass-loss 
rates and mass-loss variability of LBV winds, or that alternative mechanisms, such as pulsations or magnetic fields, need to be invoked. If radiation-pressure is the main driver, just as it is in OB-type stars, then one expects the effective temperatures at the epochs at which $\dot{M}$ has been deduced, to determine whether an increase or decrease in mass loss occurs. In such a picture, the seemingly contradictory mass loss behaviour of e.g. R 71 and R 110 may be explained in terms of their differing effective temperature intervals with respect to the bi-stability jumps (de Koter 1997; Lamers 1997).

The mass-loss rates of LBVs are expected to differ from those of normal OB stars - at the same position in the HRD as the result of two effects. First, their stellar masses (and consequently their mass over luminosity ratios) are expected to be lower as LBVs are more evolved. Second, their surface abundances are expected to be different as products of nucleosynthesis may have reached the stellar surface. Observations show that LBVs are indeed enriched in helium and nitrogen, and depleted in carbon and oxygen (e.g. Smith et al. 1994; Najarro et al. 1997). The LBV mass-loss predictions presented in this paper take these effects into account.

As LBVs have the property that their effective temperatures change substantially on timescales of years, they offer the unique opportunity to study mass loss as a function of effective temperature for individual stars. In this respect it is interesting to note that Stahl et al. (2001) have recently observed and analysed the Galactic LBV AG Carinae and found an increase in the mass-loss rate by a factor of five during its excursion from minimum to maximum visual light. Intriguingly, this increment in mass loss is similar in magnitude to the predictions of OB star mass loss around the bi-stability jump (Vink et al. 1999).

The paper is organised as follows. We first describe the Monte Carlo method used to predict mass-loss rates (Sect. 2). In Sect. 3 we present the LBV mass-loss predictions. A comparison between these theoretical mass-loss rates and all available mass-loss observations is subsequently presented in Sect. 4. Special emphasis is attributed to AG Car (Sect. 4.2), which is by far the best studied member of its group. Interestingly, the set of $\dot{M}$-determinations for this star provides such tight constraints that, in addition, the stellar mass of AG Car can be determined (Sect. 4.3). We present our conclusions in Sect. 5.

\section{Method to predict mass loss}

\subsection{A brief description of the Monte Carlo method}

We use Monte Carlo calculations of photons travelling through an atmosphere that accounts for an outflowing wind to predict mass-loss rates. In essence, the mass loss follows from equating the gain of kinetic energy of the outflowing gas to the decrease of radiative energy. From an outside observer's point of view, this decrease of radiative energy occurs when photons transfer momentum (and energy) in interactions with moving ions in the flow. An iterative process is used to find the model in which the input mass loss is equal to that found from the global radiative to kinetic energy conversion. This $\dot{M}$ value is the predicted mass-loss rate. Details of the Monte-Carlo method are given in Abbott \& Lucy (1985), de Koter et al. (1997) and Vink et al. (1999). The model atmospheres used in the process are calculated using the non-LTE code ISA-WIND and involve a gradual transition of density, consequently of velocity (through the equation of mass continuity), and temperature between the stellar photosphere and wind region. The chemical species for which the statistical equilibrium equations are solved explicitly are $\mathrm{H}, \mathrm{He}, \mathrm{C}, \mathrm{N}, \mathrm{O}$, and $\mathrm{Si}$. For a full description of the code, we refer the reader to de Koter et al. (1993, 1997). The iron-group elements, which are important for the radiative driving, are not explicitly accounted for but are treated in a generalised version of the "modified nebular approximation” described by Lucy $(1987,1999)$.

Our method to predict mass loss is distinct from the approach in which the line force is parameterised in terms of force multiplier parameters (e.g. Castor et al. 1975; Pauldrach et al. 1994). The main advantage of our approach is that it accounts for multiple photon scattering processes, which have been found to be important even for normal O- and B-type stars (e.g. Vink et al. 2000), but which are not treated in the line-force-parameterisation method. A disadvantage of our approach may be that we do not de facto solve the momentum equation, i.e. the predicted mass loss values depend to some extend on the adopted velocity stratification $v(r)$. For this we assume a $\beta$-type velocity law, which has been found to yield excellent results in modelling the spectra of hot star winds (e.g. Puls et al. 1996). We estimate the intrinsic accuracy of our Monte Carlo calculations to be $\lesssim 0.05$ dex in the predicted $\dot{M}$.

\subsection{Assumptions in the models}

Modified nebular approximation. The simplified treatment of the ionisation and excitation state of the iron-group elements, i.e. by means of the modified nebular approximation, may result in a (systematic) shift of the temperature at which the dominant ionisation state of these species changes relative to full non-LTE calculations. An indication that such an offset may indeed be present has been presented by Vink et al. (1999). These authors identify the occurrence of strong changes in the terminal velocity and ionisation of the winds of $\mathrm{OB}$ supergiants at spectral type B1 (i.e. at $21000 \mathrm{~K}$; Lamers et al. 1995) to be a result of the transition of the dominant iron ionisation from Fe IV to III. Using a similar approach as the one used in this study, they predict a jump in the mass-loss rate as a result of this recombination, but they predict the location of this jump to be at $\sim 25000 \mathrm{~K}$, i.e. at an effective temperature of $\sim 4000 \mathrm{~K}$ higher than the observed temperature of the jump. OB supergiant simulations over a wider range of wind densities indicate that the ionisation balance of Fe IV/III is mostly sensitive to temperature, and not density. Therefore, the bi-stability jump was found to occur over only a relatively narrow range of $22.5 \leq T_{\text {eff }} \leq 26 \mathrm{kK}$ (Vink et al. 2000). In other words, the offset between the predicted bi-stability jumps and the observed B1 jump was found to be in the range 1500-5500 K, with $4000 \mathrm{~K}$ being a typical value. One may therefore expect that for the dense winds of LBVs, the offset will be at least of the same magnitude as the maximum B supergiant offset, 
i.e. $5500 \mathrm{~K}$. We will later see (in Sect. 4.2) that the offset is $\simeq 6000 \mathrm{~K}$. We note that for stars that vary their effective temperatures in a range without iron recombinations, one does not expect the modified nebular approximation to affect the predicted mass loss. Indeed, in the case of O-type stars (where Fe IV is dominant over the full O-star temperature range) good agreement was found between our predictions that use the modified nebular approximations and the observed mass-loss rates (Vink et al. 2000; Benaglia et al. 2001).

In view of the above, the use of the modified nebular approximation is anticipated to cause an offset in the predicted position of the bi-stability jumps. We will apply a corrective shift to the locations of the jumps in our models to facilitate a meaningful comparison between these predictions and the observations (Sect. 4).

Stationarity. We assume a stationary stellar wind, i.e. both the mass-loss rate and the velocity stratification are timeindependent. The assumption of stationarity may not be too bad an assumption for predicting the time-averaged mass-loss rates of O stars (see e.g. Owocki et al. 1988). However, for LBVs this may not be fully correct as the timescale of photometric variability $\tau_{\text {phot }}$ becomes comparable to, or shorter than, the typical flow timescale of the wind. If we define the latter as the timescale $\tau_{\text {dyn }}$ for a gas element to travel from the photosphere to 100 stellar radii - typically the extent of the $\mathrm{H} \alpha$ formation region in LBVs - we find at minimum visual brightness $\tau_{\text {dyn }} \sim$ days, while at maximum visual light $\tau_{\text {dyn }} \sim$ months. For LBVs, significant photometric changes may occur on timescales of $\tau_{\text {phot }} \sim$ months, and therefore the assumption of a stationary wind is first expected to break down for LBVs in their transition from maximum to minimum visual light. One should realise that such a breakdown would not only occur in wind models to predict mass loss, but it would also affect the analysis of LBV spectral observations, e.g. the modelling of the $\mathrm{H} \alpha$ line profile in order to determine the mass-loss rate. A stationary model atmosphere cannot predict the line cores (which are formed at low projected velocities) and the line wings (formed at high velocities) at the same time, as this would introduce significant errors in the deduced $\dot{M}$ values.

For these reasons we focus our comparison of predictions with observations on the transition of LBVs from minimum to maximum visual light, and we do not include the reverse trajectory (see Sect. 4.2).

Sphericity. Spectropolarimetric observations of the LBV AG Car and other LBVs have shown that the winds of these stars are not spherically symmetric (see e.g. Schulte-Ladbeck et al. 1994). This may have implications for both our mass-loss predictions, as well as the modelling of the $\mathrm{H} \alpha$ lines to deduce the observed mass-loss rates. As there is little quantitative information on the density contrast between the pole and equator in LBV winds, it remains to be seen how important these nonsphericity effects are with respect to the global wind properties. In any case there is no particular reason why a possible systematic non-sphericity effect would appear more prominent in one visual brightness state than in the other.
Table 1. Adopted stellar parameters for the grid of LBV models presented in this study.

\begin{tabular}{cccc}
\hline \hline $\log L_{*}$ & $M_{*}$ & $\Gamma_{\mathrm{e}}$ & $\begin{array}{c}T_{\text {eff }} \\
(\mathrm{kK})\end{array}$ \\
\hline$\left.L_{\odot}\right)$ & $\left(M_{\odot}\right)$ & & $10.5-30.0$ \\
6.5 & $30-10$ & $0.18-0.53$ & $10.2-30.0$ \\
\hline
\end{tabular}

\subsection{The adopted parameters}

Luminosities and masses. We calculate mass-loss rates for two series of LBV models, one having a luminosity $\log \left(L / L_{\odot}\right)=5.5$ and the other 6.0. The luminosities of most LBVs fall in this range, e.g. R 71 and $\mathrm{R} 110$ are representative for the low luminosity value, while AG Car and R 127 are representative for the higher one. Since the masses of LBVs are poorly known, we adopt a range of masses for both series (see Table 1). For the series with $\log \left(L / L_{\odot}\right)=5.5$, mass loss was computed for $M=30,25,20,15,12$ and $10 M_{\odot}$. For the series with $\log \left(L / L_{\odot}\right)=6.0$, the adopted masses were $M=35$, 30 , and $25 M_{\odot}$.

The luminosity-to-mass ratio $(L / M)$ can be expressed in terms of the Eddington factor $\Gamma_{\mathrm{e}}$. This factor is defined as the ratio between the Newtonian acceleration and the radiative acceleration due to electron scattering, and is given by

$\Gamma_{\mathrm{e}}=\frac{L \sigma_{\mathrm{e}}}{4 \pi c G M}=7.66 \times 10^{-5} \sigma_{\mathrm{e}}\left(\frac{L}{L_{\odot}}\right)\left(\frac{M}{M_{\odot}}\right)^{-1}$

where the constants have their usual meaning and $\sigma_{\mathrm{e}}$ is the electron scattering cross-section per unit mass. The value for $\sigma_{\mathrm{e}}$ depends on temperature and chemical composition. The range in $\Gamma_{\mathrm{e}}$ relevant for our model grid is given in Col. 3 of Table 1. Note that $\Gamma_{\mathrm{e}}$ is considerably smaller than unity, signifying that the classical Eddington limit is not exceeded. The reason for this relatively low $\Gamma_{\mathrm{e}}$ is that although the masses are lower than for normal OB supergiants, the value for the electron scattering cross-section $\sigma_{\mathrm{e}}$ is also lower. This is a result of helium enrichment in LBV atmospheres.

Effective temperatures. Models have input temperatures computed for effective temperatures between 11000 and $30000 \mathrm{~K}$ with a stepsize of $2500 \mathrm{~K}$ in the range 12500 $30000 \mathrm{~K}$. This input temperature $T_{\text {in }}$ is defined by the relation:

$L=4 \pi R_{\text {in }}^{2} \sigma T_{\text {in }}^{4}$

where $R_{\text {in }}$ is the inner boundary of the atmospheric model. $R_{\text {in }}$ is typically located at Rosseland optical depth $20 \lesssim \tau_{\mathrm{R}} \lesssim 25$. Note that the actual effective temperature $T_{\text {eff }}$, which follows from the model computation, will be somewhat lower. The effective temperature is defined at the point where the thermalisation depth measured in the center of the $V$ band (at $5555 \AA$ ) equals $1 / \sqrt{3}$ (see Schmutz et al. 1990 and de Koter et al. 1996 for a detailed discussion). For stars with relatively low mass loss and consequently mostly optically thin winds, such as the winds from normal OB supergiants, there is no difference between the input $T_{\text {in }}$ and $T_{\text {eff. }}$ But in the case of LBVs, which 
Table 2. Solar versus adopted LBV abundances. The latter show surface helium enrichment and CNO processed material.

\begin{tabular}{cll}
\hline \hline Element & $\begin{array}{l}\text { Solar } \\
\text { (mass fraction) }\end{array}$ & $\begin{array}{l}\text { LBV } \\
\text { (mass fraction) }\end{array}$ \\
\hline $\mathrm{H}$ & 0.68 & 0.38 \\
$\mathrm{He}$ & 0.30 & 0.60 \\
$\mathrm{C}$ & $2.9 \times 10^{-2}$ & $2.9 \times 10^{-4}$ \\
$\mathrm{~N}$ & $9.5 \times 10^{-4}$ & $9.5 \times 10^{-3}$ \\
$\mathrm{O}$ & $7.7 \times 10^{-3}$ & $7.7 \times 10^{-4}$ \\
\hline
\end{tabular}

may lose mass at rates of up to $\dot{M} \sim 10^{-4} M_{\odot} \mathrm{yr}^{-1}$, there is an offset between the two temperature values, but this offset is relatively small. Note that the discrepancy between $T_{\text {in }}$ and $T_{\text {eff }}$ is more dramatic for Wolf-Rayet stars, which have much denser winds.

Abundances. Both spectroscopic analyses and models for stellar evolution show LBVs to be enriched in helium (Smith et al. 1994; Meynet et al. 1994; Najarro et al. 1997). In addition to helium enrichment, CNO processed material that has reached the surface causes an increase in the amount of nitrogen. This occurs at the cost of oxygen and carbon, which become depleted. The total amount of CNO remains nevertheless constant. The relative changes in the elements $\mathrm{CNO}$ have been adopted from the abundance computations for massive stars with rotation induced mixing by Lamers et al. (2001) and have been applied to the solar CNO abundances given by Anders $\&$ Grevesse (1989). Table 2 presents both the initial solar abundances (Col. 2) as well as the abundances modified by the CNO process (Col. 3). The metal abundance $Z$ is kept constant at the solar value, for models computed with all species included.

Velocity laws. We have calculated $\dot{M}$ for wind models with a $\beta$-type velocity law for the supersonic part of the wind:

$v(r)=v_{\infty}\left(1-\frac{R_{*}}{r}\right)^{\beta}$

where $v_{\infty}$ is the terminal flow velocity. Below the sonic point, a smooth transition from this velocity structure is made to one that corresponds to the photospheric density structure and adopted $\dot{M}$ (see de Koter et al. 1997 and Vink et al. 1999 for details). A value of $\beta=1$ was assumed in all calculations. The predicted mass-loss rates are found to be insensitive to the wind acceleration parameter when $\beta$ is in the range $0.7-1.5$ (Vink et al. 2000). We specified the terminal velocity adopting for the ratio $v_{\infty} / v_{\text {esc }}$ the values $1.3,2.0$, and 2.6. Lamers et al. (1995) found that a ratio of 2.6 for Galactic supergiants of spectral type earlier than $\mathrm{B} 1$; a ratio of 1.3 for $\mathrm{B} 1$ to $\mathrm{A} 0$ stars, and a tentative ratio of 0.7 for later spectral types.

\section{The predictions of mass loss of LBVs}

Differences in the wind properties of an LBV star and a normal O- or B-type supergiant in the same location of the HRD are expected to be the result of differences in stellar mass (specifically in $L / M$ ) and in surface chemical composition. Regarding the latter, in LBVs helium may be enriched at the surface and $\mathrm{CNO}$-cycle processed material may have appeared. This yields an increase in $\mathrm{N}$ and a decrease in $\mathrm{C}$ and $\mathrm{O}$ relative to the initial abundances. We will now discuss the difference in mass-loss rate between LBVs and OB-stars as a result of these effects.

\subsection{The effect of CNO processed material on $\dot{M}$}

Figure 1 shows a comparison in mass-loss behaviour between models with initial (solid lines) and CNO processed abundances (dashed lines). The results are very similar, i.e. the occurrence of $\mathrm{CNO}$ processed material does not change the predicted mass loss in any major way. There are two reasons for this. First, in the case of Galactic abundances (which are being discussed here) the dominant wind driving element is iron, relative to which CNO only plays a modest role in determining the mass-loss rate (Vink et al. 1999; Puls et al. 2000). Second, as the $\mathrm{CNO}$ elements only serve as a catalyst in the nuclear reactions, the total number $\mathrm{C}+\mathrm{N}+\mathrm{O}$ remains constant. The net effect of the nitrogen enrichment and the carbon/oxygen depletion on the line driving is therefore expected to be small, as it relies on differential effects in the number of lines provided by the relevant ions of these species.

\subsection{The effect of helium enrichment on $\dot{M}$}

To investigate the difference in mass-loss behaviour as a result of changes in the hydrogen and helium abundance, we compared predictions for $(X, Y)=(0.68,0.30)$ and $(0.38,0.60)$. The models have $\log \left(L / L_{\odot}\right)=6.0$ and $M=60 M_{\odot}$, and are presented in Fig. 2. The solar abundance models (dashed lines) have been taken from Vink et al. (2000). The models with enhanced helium (solid lines) are characteristic for what is expected in the LBV phase (Meynet et al. 1994).

The most pronounced difference between the two sets of models is that at $T \gtrsim 25000 \mathrm{~K}$ (for $v_{\infty} / v_{\text {esc }}=1.3$ and 2.0 ) the helium enriched models show a higher mass loss, while at lower temperatures they feature a lower mass loss. The difference in $\dot{M}$ extends up to $\sim 0.2$ dex. A second difference is that the position of the bi-stability jump at $\sim 25000 \mathrm{~K}$ (where the iron ionisation in the region of strong wind acceleration flips between Fe IV and III) appears to shift slightly towards higher temperature, while the opposite occurs for the jump at $\sim 15000 \mathrm{~K}$ (i.e. where Fe III changes over to Fe II).

The cause of this behaviour is not the result of a difference in the line driving of $\mathrm{H}$ I relative to $\mathrm{He} \mathrm{I}$ and $\mathrm{He}$ II, as hydrogen and helium are both irrelevant for the line driving force at Galactic abundances (Vink et al. 2001). As it is the iron lines that dominate the wind driving (below the sonic point), the relevant question concerns the dominant ionisation stage of iron, and how well the wavelength positions of the Fe lines match the spectral region where the bulk of the flux is emitted. The many lines of Fe IV and Fe III are however spread throughout the Lyman and Balmer continua, and do not show a particularly strong preference for either side of the Lyman jump (at $912 \AA$ ). 


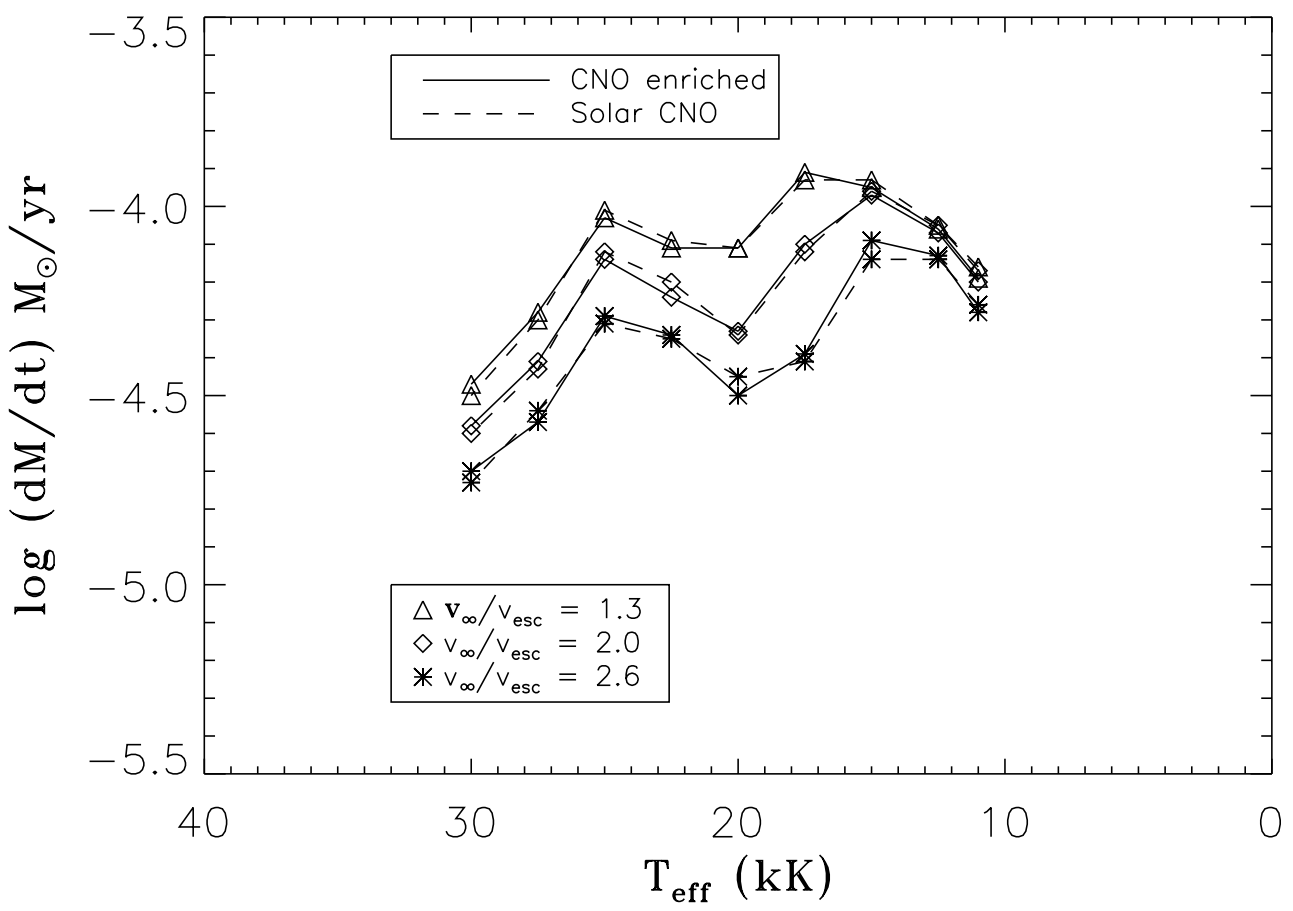

Fig. 1. Predictions of mass loss as a function of effective temperature for a star with solar (dashed lines) versus CNO processed surface abundances (solid lines). As CNO serves as a catalyst in the nuclear reactions the total number $\mathrm{C}+\mathrm{N}+\mathrm{O}$ atoms is invariant. The adopted stellar parameters are $\log \left(L / L_{\odot}\right)=6.0 ; M=30 M_{\odot} ;(X, Y)=(0.68,0.30)$. Adopted values for the ratio between terminal and effective escape velocity are given in the legend.

Therefore, the cause of the differences in mass loss behaviour must instead be related to changes in the underlying flux distribution, in particular the strength of the Lyman jump.

When hydrogen is (partly) replaced by helium, the size of the Lyman jump decreases. This implies that more photons are available in the Lyman continuum (at wavelengths below $912 \AA$ ). Since the luminosity of the models is kept constant, less flux will be emitted in the Balmer continuum. At relatively low $T_{\text {eff }}$, the bulk of the flux is emitted in the Balmer continuum, therefore one expects the mass loss in the heliumenriched models to be lower; at relatively high $T_{\text {eff }}$, most of the flux is emitted in the Lyman continuum, and as the flux in the helium-enriched models is higher here, more mass loss occurs.

The small differences in the $T_{\text {eff }}$ positions of the two bistability jumps can be explained in a similar way. In the regime of the Fe IV to III jump at $\sim 25000 \mathrm{~K}$, the wind densities in the helium-enriched models are higher, therefore recombination/ionisation will occur at a slightly higher temperature relative to the solar abundance models. For the Fe III/II jump at $\sim 15000 \mathrm{~K}$ the situation is reversed: the wind densities in the helium-enriched models are lower, therefore the jump occurs at somewhat lower $T_{\text {eff }}$.

\subsection{The effect of lower stellar masses on $\dot{M}$}

The effect of decreasing the stellar mass is shown in Fig. 3 for a set of models that have $\log \left(L / L_{\odot}\right)=5.5$. The calculations show that for all temperatures the mass loss increases with decreasing stellar mass. For this luminosity, Vink et al. (2000) derived $\log \dot{M} \propto-1.3 \log M$ for masses in the range between 30 and $50 M_{\odot}$. Here, we find that the predicted dependence is given by

$\log \dot{M} \propto-1.81( \pm 0.05) \log M$

for masses from 30 down to $10 M_{\odot}$. For this mass range, this steep dependence implies an increase of the mass loss by a factor of seven, when the mass is decreased by a factor of three (from 30 to $10 M_{\odot}$ ). This strong sensitivity can, in principle, be used as a diagnostic to derive current LBV masses. We will return to this in Sect. 4.2.

Also visible in Fig. 3 is a gradual shift of the location of the bi-stability jumps towards higher temperature for lower stellar masses. This is as one would expect: the models with low stellar mass have a relatively high mass loss, and consequently high wind density. The Saha-Boltzmann ionisation equilibrium (as formulated in the modified nebular approximation) then implies that recombination will occur at a somewhat higher temperature.

For the series with luminosity $\log \left(L / L_{\odot}\right)=6.0$ predictions for the mass-rate as a function of stellar mass are presented in Fig. 4. The calculations for 60 and $80 M_{\odot}$ shown in Vink et al. (2000) have been extended here to 35 (top panel), 30 (middle panel), and $25 M_{\odot}$ (lower panel). Results are shown for three different ratios of $v_{\infty} / v_{\text {esc }}$. The same trends are visible and the same mass-loss vs. stellar mass dependence can be inferred.

\subsection{Summary}

We conclude from the comparison between the mass loss of normal OB supergiants and LBVs that: (1) CNO processed material at the surface of LBVs does not result in a noticeable 


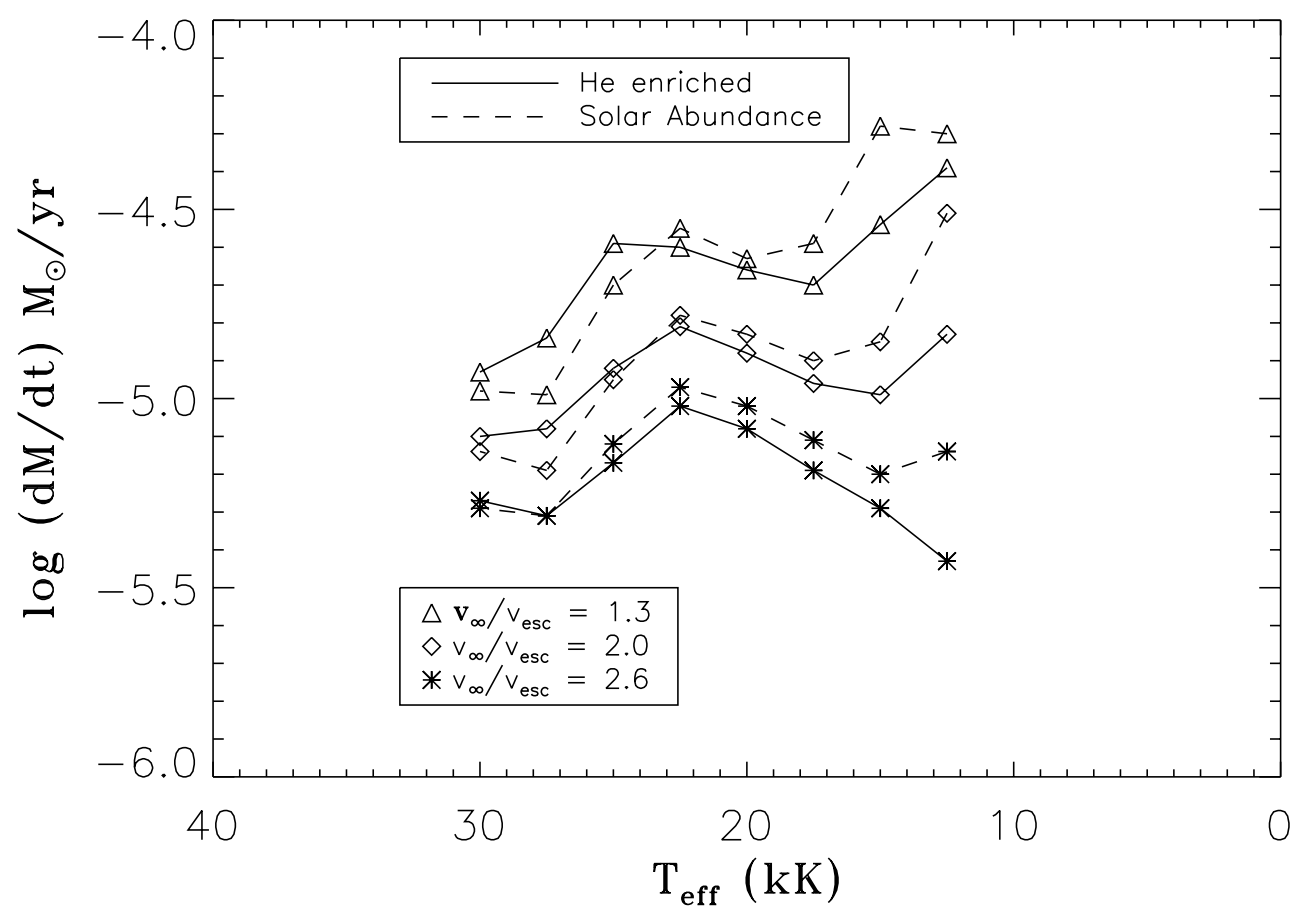

Fig. 2. Predictions of mass loss as a function of effective temperature for a star with solar (dashed lines) versus helium enriched $(X=0.38$, $Y=0.60$ ) abundances (solid lines). The adopted stellar parameters $\operatorname{are} \log \left(L / L_{\odot}\right)=6.0$ and $M=60 M_{\odot}$. Adopted values for the ratio between terminal and effective escape velocity are given in the legend.

change in $\dot{M}$ as the total number of effective driving lines is not significantly affected. (2) Helium enrichment in LBVs causes a modest change in mass loss which can be traced back to a decreased Lyman jump at $912 \AA$. (3) The reduced stellar mass of LBVs causes a strong increase in the mass-loss rate relative to normal OB supergiants.

\section{Comparison between LBV predictions and observations}

\subsection{Observed mass-loss behaviour of LBVs}

In a recent census, van Genderen (2001) identifies a total number of $34 \mathrm{LBV}$ members in the Galaxy and the Magellanic Clouds. He divides the LBVs into three groups: strong-active members, characterised by light amplitudes of $\Delta V>0.5 \mathrm{mag}$; weak-active members showing $\Delta V<0.5 \mathrm{mag}$; and ex- and dormant members which have not shown any typical photometric variability in the previous century. As our interest is in the mass loss behaviour of LBVs as a function of effective temperature, we are especially interested in the strong-active members. Although all of these 14 stars have been monitored photometrically over the last decades, only a handful have been subject to quantitative spectroscopic investigation at different epochs of photometric variability. This implies that estimates of the basic stellar parameters, such as the mass-loss rate, are rarely found at different epochs. In this respect, AG Car is by far the best investigated member, with detailed studies undertaken by Leitherer et al. (1994), Schmutz (1997) and most recently Stahl et al. (2001). We will focus on AG Car in Sect. 4.2, but we first present a brief overview of the other three LBVs that have been investigated spectroscopically. These LBVs are all in the Large Magellanic Cloud.

R71: Spectra of Radcliffe 71 at minimum and maximum visual light have been discussed in detail by Wolf et al. (1981). They derive temperatures for the two phases of $T_{\text {eff }} \sim 13600$ and $6000 \mathrm{~K}$ respectively. The luminosity of the star is about $10^{5.5} L_{\odot}$. From a simple $\mathrm{H} \beta$ line profile analysis they determine $\dot{M}=10^{-6.5} M_{\odot} \mathrm{yr}^{-1}$ for the hot phase. For the cool phase they estimate from the observed infrared excess that $\dot{M}=$ $10^{-4.3} M_{\odot} \mathrm{yr}^{-1}$. In a historical context, it is essentially this difference of a factor 100-200 that spurred the idea of explaining the radius changes of LBVs by a variable mass loss, leading to the formation of pseudo-photospheres at maximum light (Davidson 1987; Lamers 1987). However, for two reasons we suspect that the derived maximum mass loss may be incorrect. First, dust emission contributes to the near-infrared flux of R 71 (e.g. Voors et al. 1999) which Wolf et al. (1981) have not corrected for. This implies that they have overestimated $\dot{M}$. Second, the change in the $\mathrm{H} \beta$ profile between minimum and maximum light does not corroborate a change in mass loss of a factor 100-200. In case $\mathrm{H} \beta$ is optically thin, it holds that

$\frac{\dot{M}_{\text {max }}}{\dot{M}_{\min }} \propto\left(\frac{\mathcal{F}^{\mathrm{c}}{ }_{\text {max }} \mathcal{W}_{\text {max }}^{\mathrm{eq}}}{\mathcal{F}^{\mathrm{c}}{ }_{\text {min }} \mathcal{W}_{\text {min }}^{\mathrm{eq}}}\right)^{1 / 2}$

where $\mathcal{F}^{\mathrm{c}}$ denotes the continuum flux at $4861 \AA$ and $W^{\text {eq }}$ represents the equivalent width of $\mathrm{H} \beta$. The above equation assumes that the terminal velocity does not vary between minimum and maximum phase, which has indeed been found to be the case (Wolf et al. 1981). To estimate the mass loss ratio between visual minimum (min) and maximum (max) light, we use the 


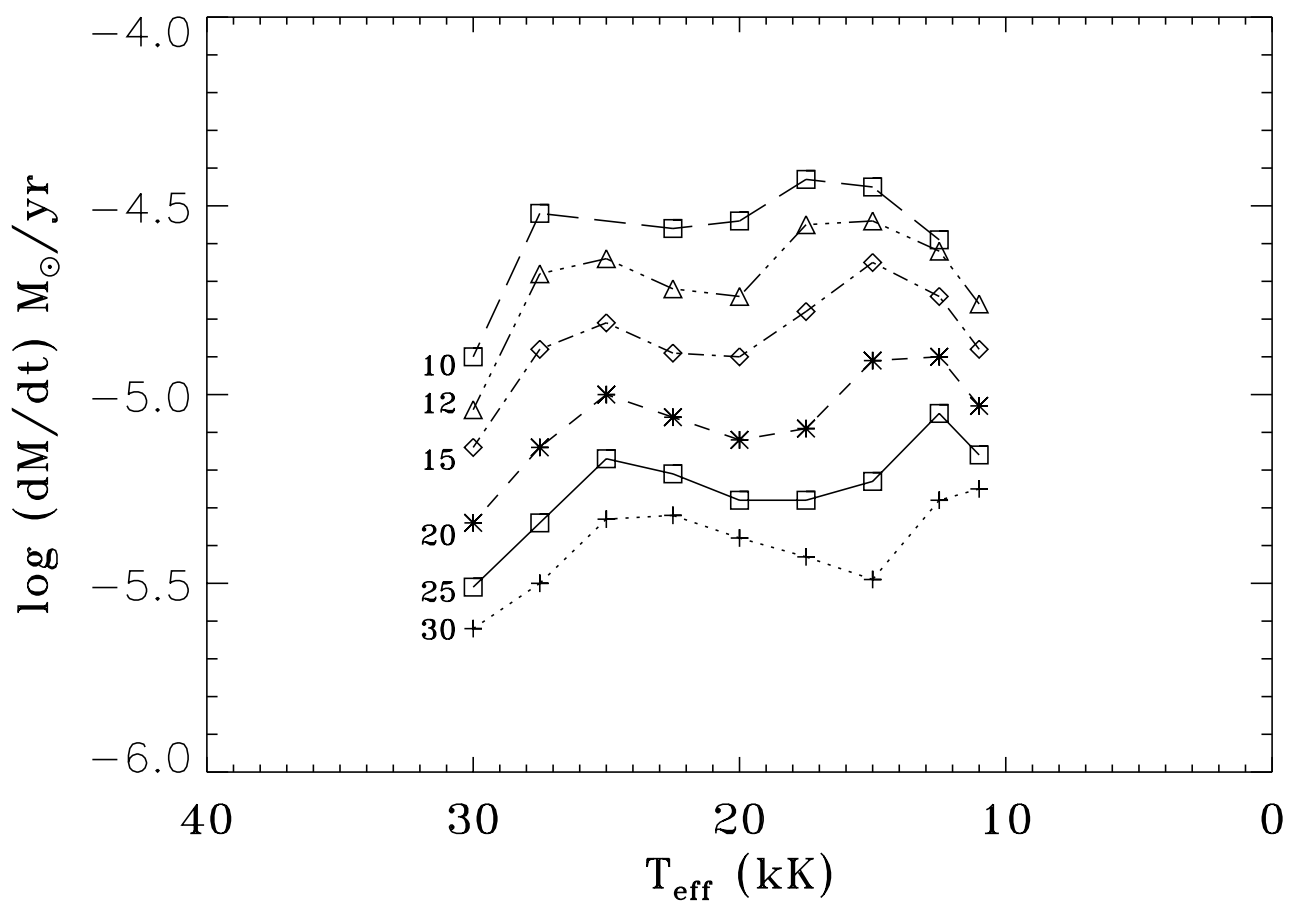

Fig. 3. Predictions of mass loss as a function of stellar mass for a star with solar abundances. The adopted luminosity is $\log \left(L / L_{\odot}\right)=5.5$. We find that $\log \dot{M} \propto-1.8 \log M$. The different masses in $M_{\odot}$ are given in the plot.

$\mathrm{H} \beta$ peak strength ratios, as given by Leitherer et al. (1989), of respectively 1.3 and 5.0 to approximate the equivalent width ratio. We estimate the continuum flux ratio using the total visual magnitude change of $\Delta V \sim 1 \mathrm{mag}$. We then arrive at a mass loss at maximum visual light of $\sim 3 \dot{M}_{\text {min }}$. In case the line would be optically thick, and the power-law index in Eq. (5) is alternatively given by $4 / 3$ (de Koter et al. 1996), we find that $\dot{M}_{\max } \sim 5.5 \dot{M}_{\min }$. Note that model calculations of Leitherer et al. (1989) also indicate that the change in the $\mathrm{H} \beta$ profile points to a much more modest difference in mass loss between the two phases than the factor 100-200 estimated by Wolf et al. (1981). We come to the conclusion that R 71 shows an increase in mass loss of a factor of 3 to 5 when it changes its temperature from $\sim 13600$ to $6000 \mathrm{~K}$. Our models indeed predict a change of this magnitude as the star crosses the Fe III to Fe II recombination jump at $10000 \mathrm{~K}$.

R 127: R 127 has been extensively monitored by the Heidelberg group (Stahl et al. 1983; Stahl \& Wolf 1986; Wolf et al. 1988). Based on spectral type determination, the stellar temperature is found to vary between about 30000 and $10000 \mathrm{~K}$. The authors note a rather capricious behaviour of the mass-loss rate. We anticipate such behaviour as two iron recombination jumps are located within this temperature range, i.e. Fe IV to III at $\sim 21000 \mathrm{~K}$ and Fe III to II at $\sim 10000 \mathrm{~K}$. Unfortunately, a lack of well determined mass-loss rates at different phases in its variability cycle prevents a quantitative comparison.

R 110: The lightcurve of $\mathrm{R} 110$ has been discussed in detail by van Genderen et al. (1997). The star has been investigated spectroscopically at two different epochs by Stahl et al. (1990). They found that in the course of brightening, when the star changed its spectrum from B-type with P Cygni lines to normal F0, the mass loss decreased from an estimated few times $10^{-6} M_{\odot} \mathrm{yr}^{-1}$ to a value that cannot be much higher than about $10^{-6} M_{\odot} \mathrm{yr}^{-1}$. The implication of the finding that $\dot{M}$ is smaller for lower $T_{\text {eff }}$ is that the cause of the excursions of LBVs in the HR-diagram cannot be the formation of an opaque stellar wind as the result of an increased mass loss. Our mass-loss predictions do indeed show $T_{\text {eff }}$ intervals for which the mass loss increases inversely proportional to the temperature. The uncertainty in the derived mass loss by Stahl et al. (1990) is however too large to allow for a meaningful quantitative comparison.

\subsection{The mass-loss behaviour of AG Car}

AGCar is by far the best observed LBV in terms of its mass-loss behaviour. Leitherer et al. (1994) derive $\dot{M}\left(T_{\text {eff }}\right)$ for the rising branch in its lightcurve covering the period from December 1990 to June 1992. Stahl et al. (2001) also investigate the mass loss behaviour of the star covering the rising and falling branches in the epoch December 1990-August 1999. The methods in these papers to determine $\dot{M}$ are to some extent rather similar. In both studies the luminosity of the star is assumed to be constant at $\log \left(L / L_{\odot}\right)=6.0$, and the temperature is derived by fitting the observed flux in the $V$-band to that of non-LTE model atmospheres (see the respective papers for details). The two methods differ in the way the mass loss is derived. Leitherer et al. used the equivalent width of $\mathrm{H} \alpha$, while Stahl et al. modelled the $\mathrm{H} \alpha$ profile at selected phases. The latter method is more accurate, and it yields more reliable $\dot{M}$ values; it additionally allows one to set constraints on the 

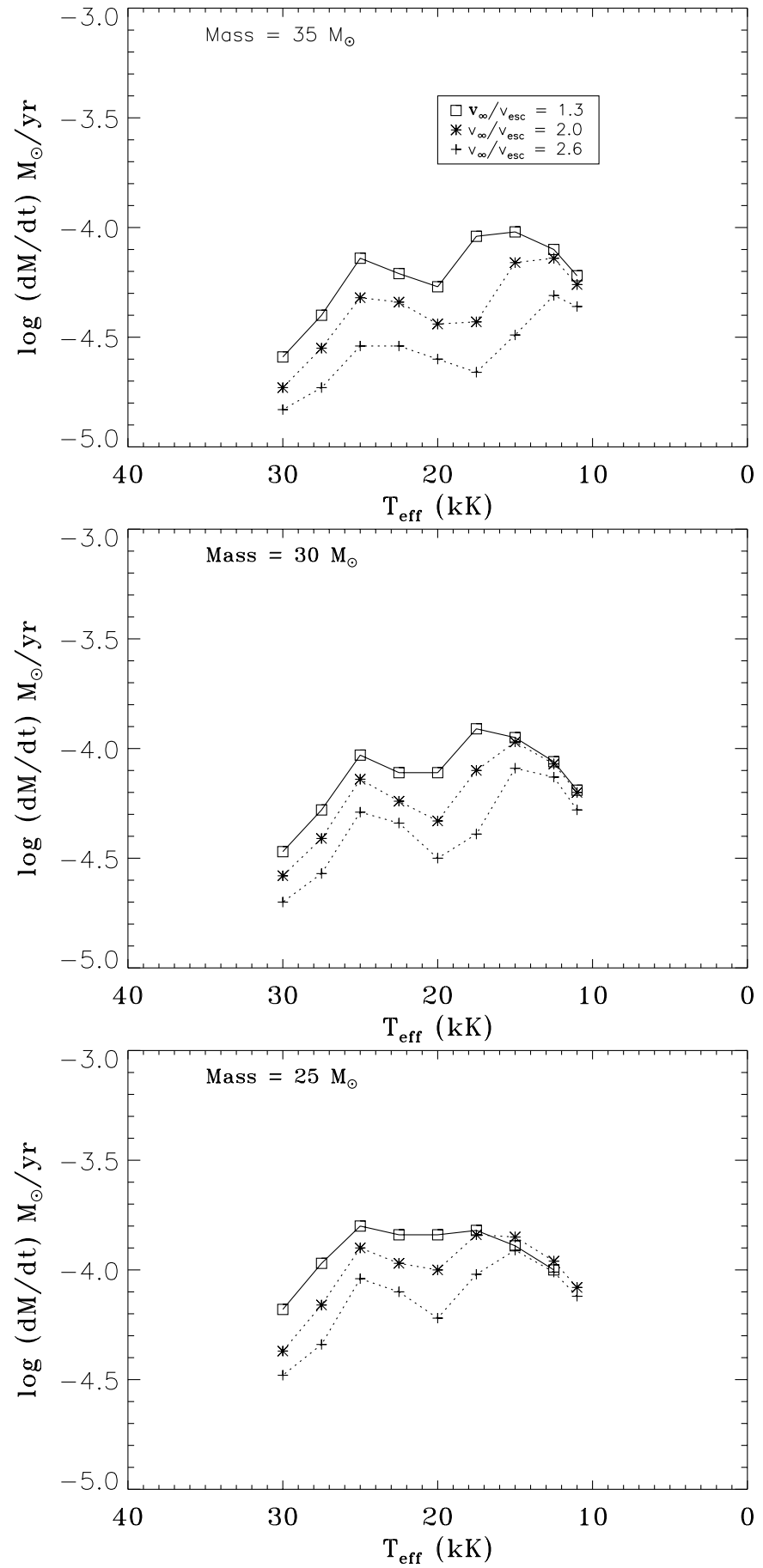

Fig. 4. Predicted mass-loss rates as a function of effective temperature for three adopted stellar masses. All models have $\log \left(L / L_{\odot}\right)=6.0$. The values for the ratio between terminal and effective escape velocity are given in the upper panel.

velocity law. We will therefore compare our predictions to the data of Stahl et al. (2001). Their derived mass-loss rates are plotted against effective temperature in Fig. 5 for both the rising (solid line) and the falling branch (dotted line). Note that there is a difference in behaviour between the star crossing over from minimum to maximum visual light, and visa versa. Our models do not predict such behaviour, as the basic stellar parameters (including stellar mass) are identical at similar positions in the

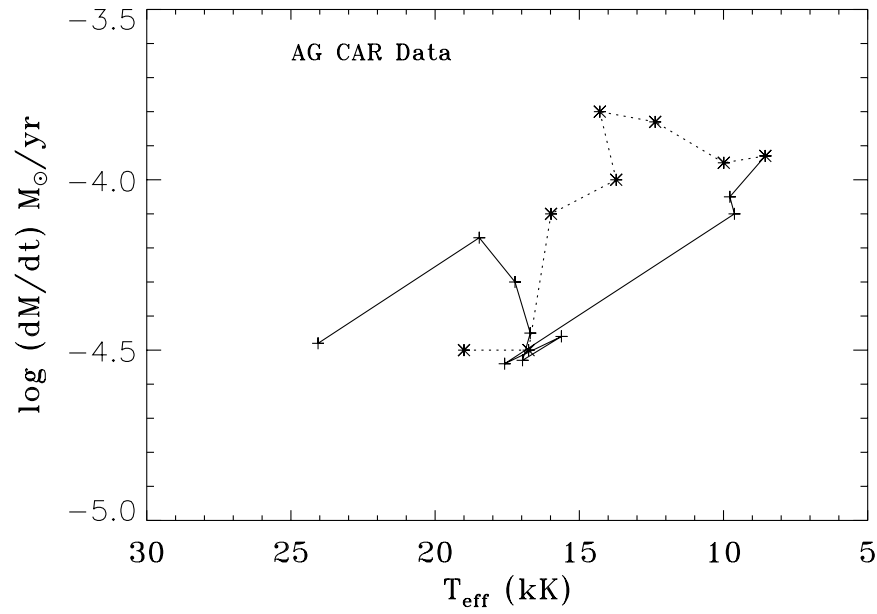

Fig. 5. Mass-loss rates of AGCar as a function of effective temperature as derived from analysis of the $\mathrm{H} \alpha$ profile by Stahl et al. (2001). The solid line connects points on a rising branch in the lightcurve, i.e. when the star is on its way from minimum to maximum visual light, and covers the epoch Dec. 1990-Feb. 1995. The dashed line indicates the mass-loss behaviour on the falling branch, covering the period Feb. 1995-Aug. 1999.

HRD on the rising and the falling branch. We suspect that the differences in $\dot{M}$ between the two branches, as derived by Stahl et al. (2001), are the result of the breakdown of the assumption of a stationary wind (see Sect. 2.2) for the falling branch. The long dynamical timescale of the flow at maximum light implies that material lost in that phase may still be in close vicinity to the star, affecting the mass loss determination in the falling branch leading to a spuriously high (surface) mass-loss rate. A second explanation may be the release of gravitational energy when the star returns from maximum to minimum. If this plays a role, the assumption of constant bolometric magnitude during the variability is no longer valid (see Lamers et al. 1995). Whether or not AG Car remains at constant luminosity during its typical variations has however not yet been firmly established. Because of the two above-mentioned complications, we will focus the comparison of our predictions to the observed mass-loss rates to the rising branch only.

In Fig. 6 we compare our mass-loss predictions for AG Car with the rates determined by Stahl et al. (2001) for the rising branch over the epoch Dec. 1990-Aug. 1999, where the temperature decreases from 24000 to $9000 \mathrm{~K}$. In our calculations we adopt $\log \left(L / L_{\odot}\right)=6.0$; a stellar mass $M=35 M_{\odot}$; a helium mass fraction $Y=0.60$, and a constant ratio of terminal over escape velocity $v_{\infty} / v_{\text {esc }}=1.3$. The luminosity and helium abundance are very similar to that assumed by Stahl et al. (2001). The stellar mass is a free parameter or, phrased differently, fitting the mass loss allows one to determine the mass (see Sect. 4.3). Unfortunately, the ratio $v_{\infty} / v_{\text {esc }}$ turns out to be rather poorly constrained by observations, as $\mathrm{H} \alpha$ only allows to determine a lower limit to $v_{\infty}$ (Stahl et al. 2001). Taking these lower limits and correcting the escape velocity for electron scattering, Stahl et al. find lower limits in the range $0.2 \lesssim v_{\infty} / v_{\text {esc }} \lesssim 1.1$. We have opted to use our lowest calculated $v_{\infty} / v_{\text {esc }}$ for the whole temperature range, as this is the most 


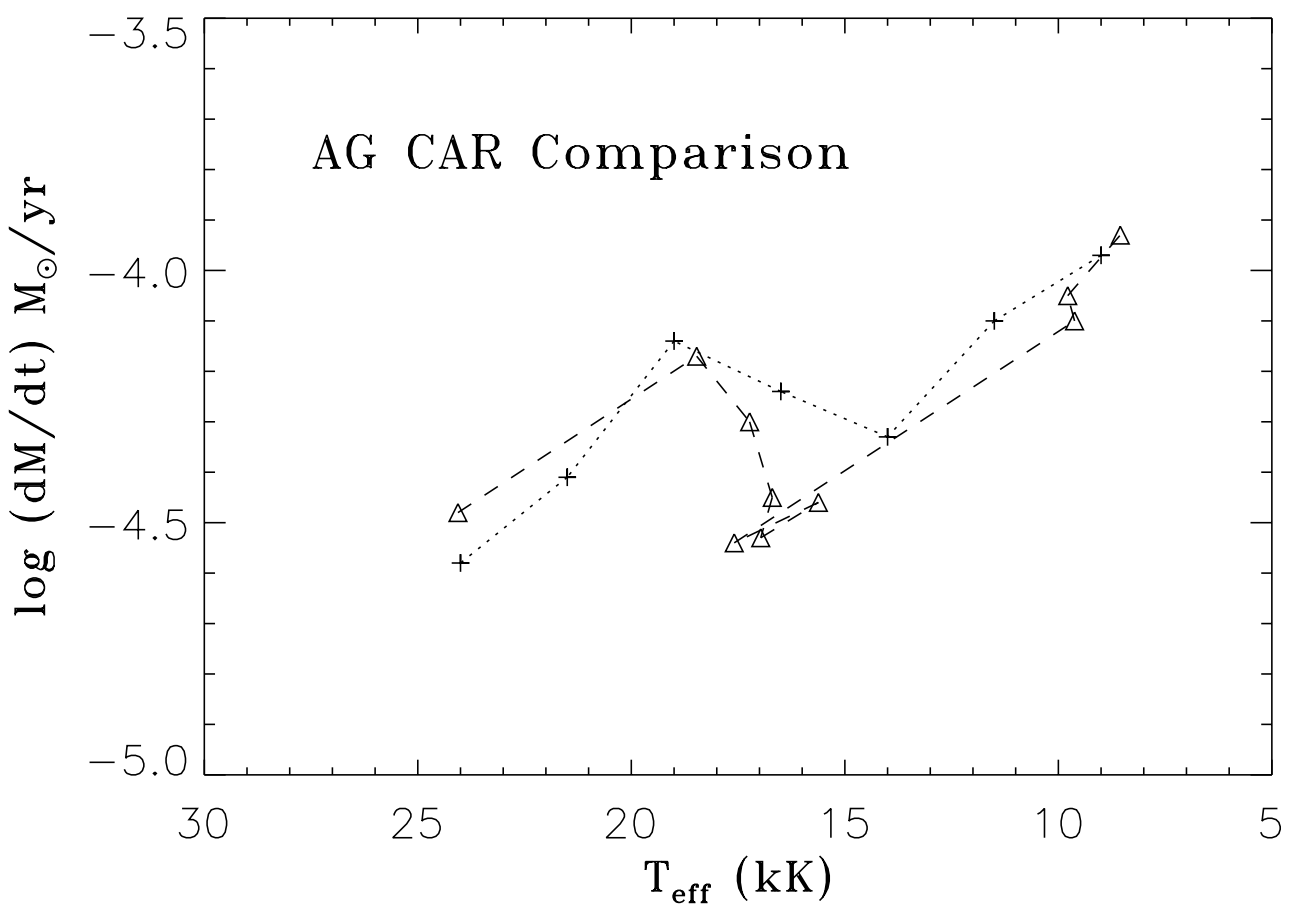

Fig. 6. Comparison of the predicted (dotted line) and observed (dashed line) mass loss of AG Car. The adopted stellar parameters are $\log \left(L / L_{\odot}\right)=6.0 ;(X, Y)=(0.28,0.60)$, and $v_{\infty} / v_{\text {esc }}=1.3$. All calculations have been shifted by $\Delta T_{\text {eff }}=-6000 \mathrm{~K}$ to correct for a systematic offset in the predicted ionisation balance of iron. The good agreement allows us to determine the present-day mass of AG Car to be $35 M_{\odot}$ by fitting the mass-loss rates.

appropriate value. We need to make two remarks with respect to this choice.

First, the adopted ratio of the terminal over the effective escape velocity influences the present-day mass determination of the star. For instance, if we would adopt a value $v_{\infty} / v_{\mathrm{esc}}=$ 2.0 we would find $M=30 M_{\odot}$, while a choice of $v_{\infty} / v_{\text {esc }}=$ 2.6 would yield $M=25 M_{\odot}$. To first order, this positive relation between the velocity ratio and the stellar mass is explained as follows. For fixed stellar parameters, the photon momentum $(L / c)$ transfered to the wind $\left(\dot{M} v_{\infty}\right)$ is more or less constant. It would therefore be necessary to decrease the stellar mass to match the observed mass-loss rates. However, if the actual value of $v_{\infty} / v_{\text {esc }}$ were less than 1.3 , we would anticipate that the mass would be lower than $35 M_{\odot}$ rather than higher. This because Doppler shifting of lines is strongly reduced for low velocity winds, which decreases the effectiveness of wind driving and would indeed require a lower stellar mass to match the observed $\dot{M}$.

Second, the temperature range investigated by Stahl et al. (2001) includes the regime where iron recombines from Fe III to Fe II. As a result of this, we predict a jump in mass loss around $15000 \mathrm{~K}$. Possibly, this jump is related to the drop in the ratio $v_{\infty} / v_{\text {esc }}$ from about 1.3 to 0.7 at spectral type A0, as identified by Lamers et al. (1995) on the basis of observed terminal velocities of supergiants. Because A0 I stars have $T_{\text {eff }} \sim$ $10000 \mathrm{~K}$, a similar shift between predicted and observed ionisation appears to be present as was found for the recombination of Fe IV to Fe III around spectral type B1 (cf. Sect. 2.2). In order to compensate for this offset, we shift our calculations by $\Delta T_{\text {eff }}=-6000 \mathrm{~K}$. The observed change in terminal velocity at spectral type A0 would affect the predicted $\dot{M}$ for the points at visual maximum light (i.e. at $T_{\text {eff }} \lesssim 10000 \mathrm{~K}$ ). As this is at the very low end of the investigated temperature range, we have opted not to decrease $v_{\infty} / v_{\text {esc }}$ further, i.e. below 1.3.

Figure 6 shows that after accounting for the corrective shift $\Delta T_{\text {eff }}$, the observed and predicted mass loss agree within about 0.1 dex. Taking into account that $\dot{M}\left(T_{\text {eff }}\right)$ shows a complex behaviour, fluctuating over more than $0.5 \mathrm{dex}$, this is a surprisingly good result. It shows that the mass loss variability of AG Car is the result of changes in the ionisation of the dominant line-driving element, iron.

\subsection{The stellar mass of AG Car}

The $\dot{M}\left(T_{\text {eff }}\right)$ behaviour can also be used to constrain the stellar mass. The reason is that the predicted mass-loss rates are not only a function of $T_{\text {eff }}$, but also of stellar mass (see Eq. (4)). Comparing the AG Car data with $\dot{M}\left(T_{\text {eff }}\right)$ predictions for different masses results in a best fit for the present-day mass of $35 \pm 5 M_{\odot}$. Note that this mass estimate does not account for possible systematic errors. Notably, one should be aware that the $\mathrm{H} \alpha$ spectral modelling procedure of Stahl et al. (2001) did not account for clumping. In the case of Wolf-Rayet stars it is well-established that the neglect of a clumping correction may lead to systematic errors in the mass-loss rates by factors of $\sim 2-4$ (see e.g. Hillier 2000). If clumping also plays an important role in the winds of LBVs, this would imply that the massloss rates derived by Stahl et al. are too high. Consequently, our mass derivation would be underestimated. We will now discuss 
whether a present-day mass of $35 M_{\odot}$ is a reasonable value for AG Car.

Applying multiple distance indicators, Humphreys et al. (1989) constrain the distance to AG Car to be 5-7 kpc, implying a luminosity of $\log \left(L / L_{\odot}\right)=6.22 \pm 0.16$. Comparison with evolutionary tracks (Schaller et al. 1992) indicates that the most appropriate initial mass for such a large luminosity equals $120 M_{\odot}$ (but it would be only $85 M_{\odot}$ when main sequence mass loss is taken twice the canonical value; Meynet et al. 1994). The extreme brightness of such a star would imply a very high mass loss, which affects the evolution in a profound way. The evolutionary tracks show that a star with an initial mass of $120 M_{\odot}$ evolves to the Wolf-Rayet phase almost directly from the main sequence, without even entering a postmain sequence supergiant phase. This scenario is very difficult to reconcile with the (range of) spectroscopic properties observed for AG Car, which indicates that its luminosity must be less than $\log \left(L / L_{\odot}\right)=6.22$. For these reasons, we adopt $\log \left(L / L_{\odot}\right)=6.0$, a value which is still in fair agreement with the lower bound found by Humphreys et al. (1989). The corollary of all this is that the initial mass for AG Car is only $65 M_{\odot}$, if we assume that the star is indeed in a post-main-sequence phase.

Voors et al. (2000) have determined the mass of the dust shell around AG Car and arrived at $0.25 M_{\odot}$, with an accuracy of about a factor of two. This error is dominated by the uncertainty in the maximum size of the dust grains. Adopting the usual gas/dust ratio of 100, one finds a total circumstellar mass of about $25 M_{\odot}$. If we assume that this material is of stellar origin, which is supported by the enhanced $N$ abundance found in the ionised part of the nebula (Hutsemékers \& van Drom 1991; Smith et al. 1998), the upper limit to the present-day mass of the star is $\sim 40 M_{\odot}$. This is in good agreement with the mass of $35 M_{\odot}$ that we have derived in this study.

\section{Summary and conclusions}

We have presented radiation-driven wind models for a set of stellar masses and luminosities characteristic for LBVs and have predicted their mass-loss rates. We studied the effects of lower masses and modified abundances on the mass-loss rates of LBVs in comparison to normal OB supergiants. We have found that the main difference is due to the lower masses of LBVs. In addition, we have found that an increased helium abundance changes the mass loss properties by small amounts (of up to about $0.2 \mathrm{dex}$ in $\log \dot{M}$ ), while CNO processing is relatively unimportant for the mass-loss rate.

The comparison of our predictions with the observed massloss behaviour of LBVs has shown that:

1. The dominant driving mechanism of LBV winds is radiation pressure on spectral lines. Other mechanisms are expected to play only a minor role.

2. The observed variable mass loss behaviour of LBVs of up to $\sim 0.5$ dex during their $\mathrm{S}$ Doradus-type variation cycles can be explained in terms of changes in the efficiency of line driving, as a result of the recombination/ionisation of $\mathrm{Fe}$ IV/III and Fe III/II.
3. The $\dot{M}\left(T_{\text {eff }}\right)$ behaviour of AG Car can be matched to within 0.1 dex when a shift in temperature of $\Delta T_{\text {eff }}=-6000 \mathrm{~K}$ is applied to our predictions. This shift is required to correct for an inaccurate calculation of the ionisation balance of iron, as we have used a modified nebular approximation for this element. Note that the shift is consistent with constraints set by observations of supergiants which indicate that the Fe IV to III and the Fe III to II recombinations indeed occur at spectral types B1 and A0 (Lamers et al. 1995).

4. We have deduced a value of $35 M_{\odot}$ for the present-day mass of AG Car. If we adopt a luminosity of $10^{6} L_{\odot}$ (which implies an initial mass of $\sim 65 M_{\odot}$ ) and correct for the amount of ejected material found in the circumstellar environment of the star ( $25 M_{\odot}$; Voors et al. 2000), we arrive at a present-day mass upper limit of $\sim 40 M_{\odot}$, which is consistent with our finding of $35 M_{\odot}$.

Acknowledgements. We thank Henny Lamers for many fruitful discussions. JV acknowledges financial support from the Dutch NWO Council for Physical Sciences, the Particle Physics and Astronomy Research Council of the United Kingdom, and the Dutch research school for astrophysics (NOVA) for a travel grant. AdK acknowledges support from NWO Pionier grant 600-78-333 to L. B. F. M. Waters and from NWO Spinoza grant 08-0 to E. P. J. van den Heuvel.

\section{References}

Abbott, D. C., \& Lucy, L. B. 1985, ApJ, 288, 679

Anders, E., \& Grevesse, N. 1989, GeCoA, 53, 197

Benaglia, P., Cappa, C. E., \& Koribalski, B. S., A\&A, 372, 952

Castor, J. I., Abbott, D. C., \& Klein, R. I. 1975, ApJ, 195, 157

Davidson, K. 1987, ApJ, 317, 760

de Koter, A. 1997, in Luminous Blue Variables: Massive Stars in Transition, ed. A. Nota, \& H. J. G. L. M. Lamers, ASP Conf. Ser., 83,66

de Koter, A., Schmutz, W., \& Lamers, H. J. G. L. M. 1993, A\&A, 277, 561

de Koter, A., Lamers, H. J. G. L. M., \& Schmutz, W. 1996, A\&A, 306, 501

de Koter, A., Heap, S. R., \& Hubeny, I. 1997, ApJ, 477, 792

Hillier, D. J. 2000, in Thermal and Ionization Aspects of Flows from Hot Stars, ed. H. J. G. L. M. Lamers, \& A. Sapar, ASP Conf. Ser., 204, 161

Humphreys, R. M., \& Davidson, K. 1994, PASP, 106, 1025

Humphreys, R. M., Lamers, H. J. G. L. M., Hoekzema, N., \& Cassatella, A. 1989, A\&A, 218, L17

Hutsemékers, D., \& van Drom, E. 1991, A\&A, 251, 620

Lamers, H. J. G. L. M. 1987, in Instabilities in Luminous Early-Type Stars, ed. H. J. G. L. M. Lamers, \& C. W. H. de Loore, ASSL, 157, 99

Lamers, H. J. G. L. M. 1995, in Astrophysical applications of stellar pulsation, ed. R. S. Stobie, \& P. A. Whitelock, ASP Conf. Ser., 83,176

Lamers, H. J. G. L. M. 1997, in Luminous Blue Variables: Massive Stars in Transition, ed. A. Nota, \& H. J. G. L. M. Lamers, ASP Conf. Ser., 83, 76

Lamers, H. J. G. L. M., Snow, T. P., \& Lindholm, D. M. 1995, ApJ, 455, 269

Lamers, H. J. G. L. M., Nota, A., Panagia, N., Smith, L. J., \& Langer, N. 2001, ApJ, 551, 764

Leitherer, C. 1997, in Luminous Blue Variables: Massive Stars in Transition, ed. A. Nota, \& H. J. G. L. M. Lamers, ASP Conf. Ser., 83,58 
Leitherer, C., Schmutz, W., Abbott, D. C., Hamann, W.-R., \& Wessolowski, U. 1989, ApJ, 346, 919

Leitherer, C., Allen, R., Altner, B., et al. 1994, ApJ, 428, 292

Lucy, L. B. 1987, in ESO Workshop on the SN 1987A, Proc. (A88-35301 14-90), 417

Lucy, L. B. 1999, A\&A, 345, 211

Meynet, G., Maeder, A., Schaller, G., Schaerer, D., \& Charbonel, C. 1994, A\&AS, 103, 97

Najarro, F., Hillier, D. J., \& Stahl, O. 1997, A\&A, 326, 1117

Nota, A., \& Lamers, H. J. G. L. M. 1997, Luminous Blue Variables: Massive Stars in Transition, ASP Conf. Ser., 83

Owocki, S. P., Castor, J. I., \& Rybicki, G. B. 1988, ApJ, 335, 914

Puls, J., Kudritzki, R.-P., \& Herrero, A. 1996, A\&A, 305, 171

Puls, J., Springmann, U., \& Lennon, M. 2000, A\&AS, 141, 23

Schaller, G., Schaerer, D., Meynet, G., \& Maeder, A. 1992, A\&AS, 96, 269

Schulte-Ladbeck, R. E., Clayton, G. C., Hillier, D. J., Harries, T. J., \& Howarth, I. D. 1994, ApJ, 429, 846

Schmutz, W. 1997, in Luminous Blue Variables: Massive Stars in Transition, ed. A. Nota, \& H. J. G. L. M. Lamers, ASP Conf. Ser., 83,143

Schmutz, W., Abbott, D. C., Russell, R. S., Hamann, W.-R., \& Wessolowski, U. 1990, ApJ, 355, 255
Smith, L. J., Crowther, P. A., \& Prinja, R. K. 1994, A\&A, 281, 833

Smith, L. J., Nota, A., Pasquali, A., et al. 1998, ApJ, 503, 278

Stahl, O., \& Wolf, B. 1986, A\&A, 154, 243

Stahl, O., Wolf, B., Klare, G., et al. 1983, A\&A, 127, 49

Stahl, O., Wolf, B., Klare, G., Juettner, A., \& Cassatella, A. 1990, A\&A, 228, 379

Stahl, O., Jankovics, I., Kovács, J., et al. 2001, A\&A, 375, 54

van Genderen, A. M. 2001, A\&A, 366, 508

van Genderen, A. M., de Groot, M., \& Sterken, C. 1998, A\&AS, 124, 517

Vink, J. S., de Koter, A., \& Lamers, H. J. G. L. M. 1999, A\&A, 350, 181

Vink, J. S., de Koter, A., \& Lamers, H. J. G. L. M. 2000, A\&A, 362, 295

Vink, J. S., de Koter, A., \& Lamers, H. J. G. L. M. 2001, A\&A, 369, 574

Voors, R. H. M., Waters, L. B. F. M., Morris, P. W., et al. 1999, A\&A, 341, L67

Voors, R. H. M., Waters, L. B. F. M., de Koter, A., et al. 2000, A\&A, 356, 501

Wolf, B., Appenzeller, I., \& Stahl, O. 1981, A\&A, 103, 94

Wolf, B., Stahl, O., Smolinski, J., \& Casatella, A. 1988, A\&AS, 74, 239 